\title{
Alergias alimentarias en pediatría: frecuencia, características clínicas y alergenos más frecuentes en pacientes de un consultorio pediátrico de alergia de referencia
}

\section{Food allergies in pediatrics: Frequency, clinical characteristics and most frequent allergens in patients at a pediatric referral allergy clinic}

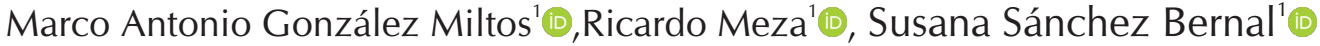 \\ ${ }^{1}$ Universidad Nacional de Asunción, Facultad de Ciencias Médicas, Catedra y Servicio de Pediatría. San \\ Lorenzo, Paraguay.
}

\section{RESUMEN}

Introducción: Las alergias alimentarias constituyen una patología cada vez más frecuente en la infancia. Es importante conocer sus características para su prevención y mejor abordaje terapéutico. Objetivo: Determinar la frecuencia, las características clínicas y los tipos de alimentos desencadenantes de las alergias alimentarias más frecuentes, en pacientes pediátricos en un consultorio de referencia, entre marzo 2016-marzo de 2019. Materiales y Métodos: estudio transversal, descriptivo, observacional con datos secundarios de fichas clínicas de pacientes de menores de 18 años. Se estudiaron edad, sexo, manifestaciones clínicas y tipo de alergeno alimentario desencadenante, detectado por RAST. Resultados: La mediana de edad de diagnóstico fue de 10 meses, fueron lactantes 52(73\%) pacientes, 10(14\%) fueron prescolares y el resto escolares y adolescentes; $43(60 \%)$ fueron varones. Las lesiones cutáneas estuvieron presentes (aisladas o combinadas con otros síntomas) en 46 casos (64\%); los síntomas respiratorios en 38 casos (53\%); gastrointestinales en 30 casos $(42,2 \%)$. La leche fue el alergeno alimentario detectado en 52 (73\%) pacientes, el huevo en 33 $(46 \%)$, el trigo en $7(9,8 \%)$, la soja en $6(8,5 \%)$, el gluten en $5(7 \%)$; las golosinas, el maní, el maíz y los embutidos en 2 $(2,8 \%)$ niños y finalmente la sandía, el tomate y los condimentos en $1(1,4 \%)$ caso. Se registró un único alérgeno en 46 casos $(64,7 \%)$, dos alérgenos en 16 casos $(22,5 \%)$, tres

\section{ABSTRACT}

Introduction: Food allergies are an increasingly frequent pathology in childhood. In order to prevent them and to offer a better therapeutic approach, it is important to characterize them. Objective: To determine the frequency, clinical characteristics and types of foods that trigger the most frequent food allergies, in pediatric patients at a referral clinic, between March 2016-March 2019. Materials and Methods: This was a cross-sectional, descriptive, observational study with secondary data from clinical records of patients under 18 years of age. Age, sex, clinical manifestations and type of triggering food allergen, detected by RAST, were studied. Results: The median age of diagnosis was 10 months, 52 (73\%) patients were infants, $10(14 \%)$ were preschool and the rest were schoolchildren and adolescents; $43(60 \%)$ were male. Skin lesions were present (isolated or combined with other symptoms) in 46 cases (64\%); respiratory symptoms in 38 cases (53\%); gastrointestinal in 30 cases (42.2\%). Milk was the food allergen detected in $52(73 \%)$ patients, egg in 33 $(46 \%)$, wheat in $7(9.8 \%)$, soy in $6(8.5 \%)$, gluten in $5(7 \%)$; sweets, peanuts, corn and sausages in $2(2.8 \%)$ children and finally watermelon, tomato and condiments in 1 $(1.4 \%)$ case. A single allergen was recorded in 46 cases $(64.7 \%)$, two allergens in 16 cases $(22.5 \%)$, three allergens in 5 cases $(7 \%)$, and more than three allergens in 4 cases

Correspondencia: Susana Sánchez Bernal, correo: susana.sanchez.ber@gmail.com

Conflicto de interés: los autores declaran no poseer conflicto de interés

Fuente de financiamiento: fondos propios

Recibido: 25/10/2021 Aceptado: 19/11/2021

DOI: https://doi.org/10.31698/ped.48032021006

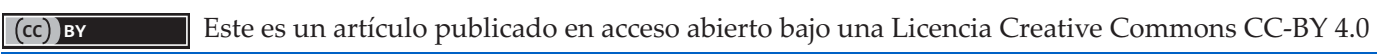


alérgenos en 5 casos (7\%), y más de tres alérgenos en 4 casos $(5,6 \%)$. Conclusiones: Los lactantes son los más afectados por las alergias alimentarias siendo más prevalentes los síntomas cutáneos y digestivos. La proteína de leche de vaca es el alergeno más frecuente seguido por el huevo.

Palabras claves: Alergia alimentaria, alergenos alimentarios, niños, pediatría.

\section{INTRODUCCIÓN}

La alergia alimentaria (AA) se define como un efecto adverso para la salud derivado de una respuesta inmune específica, que ocurre con la exposición a un alimento dado ${ }^{(1)}$. Se desarrolla debido a la inducción fallida de la tolerancia tras la exposición inicial al antígeno alimentario ${ }^{(2)}$.

La alergia alimentaria infantil se asocia con una calidad de vida deteriorada, interacciones sociales limitadas, condiciones alérgicas comórbidas y un costo económico significativo ${ }^{(3)}$ y su prevalencia varía entre 4 y $8 \%{ }^{(4)}$.

Se describe a la leche de vaca como principal desencadenante de alergias alimentarias en numerosas regiones del mundo y en la región ${ }^{(5)}$.

A menudo, otras causas de reacciones adversas a los alimentos pueden malinterpretarse como una alergia alimentaria. La intolerancia a la lactosa es un ejemplo: La incapacidad para digerir el azúcar lactosa produce meteorismo y diarrea, pero no es una alergia. Las reacciones adversas a los alimentos pueden también ser causadas por factores metabólicos, farmacológicos o tóxicos que deben distinguirse de la alergia ${ }^{(6)}$.

Entre el 35\% y $71 \%$ de las personas diagnosticadas con alergia alimentaria padecen de dermatitis atópica; entre el $33 \%$ y el $40 \%$, rinitis alérgica y entre el $34 \%$ y $49 \%$, se manifiestan como asma. La dermatitis alérgica y la alergia alimentaria coexisten frecuentemente en aquellos pacientes con dermatitis atópica de comienzo precoz, intensa y persistente; con propensión a cuadros severos, lo cual podría estar relacionado con aspectos genéticos ${ }^{(7,8,9)}$.
(5.6\%). Conclusions: Infants are the most affected by food allergies, skin and digestive symptoms are more prevalent. Cow's milk protein is the most common allergen followed by eggs.

Keywords: Food allergy, food allergens, children, pediatrics.

Es sabido que algunas alergias alimentarias infantiles mediadas por IgE, como las alergias a la proteína de la leche de vaca y al huevo, tienen más probabilidades de resolverse con una adecuada intervención con dieta de exclusión en comparación con otras, como las alergias al maní y otros frutos $\operatorname{secos}^{(10)}$. De ahí la importancia de realizar tempranamente el diagnóstico a fin de favorecer la remisión según cada caso y mejorar el pronóstico en cuanto a estas atopias. Es sabido que la mejor estrategia es la prevención y la detección oportuna de factores de riesgo ${ }^{(11)}$.

El objetivo de este trabajo fue determinar la frecuencia, las características clínicas y los tipos de alimentos asociados a las alergias alimentarias, en pacientes pediátricos menores de 18 años que acudieron al Consultorio ambulatorio de Alergia en la Cátedra y Servicio de Pediatría, en un periodo de 3 años.

\section{MATERIALES Y MÉTODOS}

Estudio observacional, descriptivo, de corte transverso y retrospectivo, en base a datos secundarios obtenidos a partir de las fichas clínicas de pacientes pediátricos, menores de 18 años, que acudieron al Consultorio de Especialidades del Hospital de Clínicas de la Universidad Nacional de Asunción. Fueron incluidos todos los niños/as y adolescentes con diagnóstico de alergia alimentaria realizado con la prueba de Radio Allergo Sorbent Test ${ }^{\circledR}$, o RAST $®$ que fue considerada como positiva con valores por encima de $0.35 \mathrm{kUa} / \mathrm{L}$.

Fueron incluidos los pacientes de ambos sexos que fueron evaluados durante el periodo comprendido 
entre el 01 de marzo del 2016 al 31 de marzo del 2019 con RAST positivo para alergenos alimentarios, por lo tanto, solo se incluyeron casos de alergia IgE mediados. Fueron excluidos pacientes con diagnóstico de patología gastrointestinal concomitante (Ej. Enfermedad Celiaca, Síndrome de intestino irritable, Enfermedad de Crohn, Colitis Ulcerosa, entre otras) y con fichas clínicas incompletas.

El muestreo fue no probabilístico por conveniencia de casos consecutivos que cumplieron con los criterios de inclusión.

Como variables fueron evaluadas: edad de diagnóstico, sexo, características clínicas detectadas y tipo de alergeno desencadenante detectado por RAST.

Se accedió al Archivo Central del Hospital de Clínicas previa autorización de la Jefatura de Cátedra y Servicio de Pediatría y se seleccionaron las fichas clínicas de pacientes a ser incluidos, correspondientes al periodo de estudio.

Los datos fueron sistematizados en el programa Excel de Microsoft Office 2010 y luego procesados con paquete estadístico Epi Info 7.

Las variables continuas se presentan en promedio o mediana, según homogeneidad de varianza como medida de tendencia central y con desvío estandar o en cuartiles como medidas de dispersión. Las variables categóricas o cualitativas se presentan en frecuencia y porcentajes.

Por la naturaleza del estudio, no se realizó el consentimiento informado sin embargo éticamente se cuidó el respeto a la identidad ya que las fichas clínicas de pacientes fueron analizadas en forma confidencial, salvaguardando su identidad, así como también fueron considerados los principios de beneficencia y no maleficencia pues no hubo perjuicio de los pacientes ni a sus familiares junto con el de justicia.

\section{RESULTADOS}

De 875 pacientes pediátricos que consultaron durante el periodo estudiado, $71(8,1 \%)$ de ellos tenían el diagnóstico de Alergia alimentaria.
En cuanto a la procedencia, 9/71 (12\%) correspondieron a Asunción, 55/71 (77,4\%) al Departamento Central, y el resto en menor proporción, provenían de otros departamentos del país (Tabla 1 ).

La mediana de edad fue de 12 meses, la edad mínima fue de 1 mes y la máxima de 16 años. Al evaluar únicamente al grupo de lactantes, la edad media al momento de la consulta fue de 10 meses ( 1 a 23 meses). Como se observa en la Tabla 1, el grupo etáreo más afectado fue el que correspondía a los lactantes 52 (73\%), 10 (14\%) fueron pre-escolares, seguido en menor proporción por los pacientes en edad escolar y adolescentes.

Tabla 1. Procedencia y aspectos demográficos de pacientes pediátricos con diagnóstico de alergia alimentaria del Hospital de Clínicas FCM-UNA Años 2006-2009.

\begin{tabular}{lcc}
\hline \multicolumn{1}{c}{ Edad (meses) } & 12 Mínimo 1, Máximo 192 \\
\hline \multicolumn{1}{c}{ Procedencia } & $\mathbf{n}$ & $\mathbf{\%}$ \\
\hline Central & 55 & 77,5 \\
Capital & 9 & 12,7 \\
Guairá & 2 & 2,8 \\
Caaguazú & 1 & 1,4 \\
San Pedro & 1 & 1,4 \\
Paraguari & 2 & 2,8 \\
Cordillera & 1 & 1,4 \\
\hline \multicolumn{1}{c}{ Sexo } & & \\
\hline Femenino & 28 & 39,4 \\
Masculino & 43 & 60,6 \\
\hline$\quad$ Grupos etáreos & & 73,2 \\
\hline Lactantes (0-23 meses) & 52 & 14,1 \\
Prescolares (24-59 meses) & 10 & 7,0 \\
Escolares (60-119 meses) & 5 & 5,6 \\
Adolescentes ( $\geq 10$ años) & 4 & \\
\hline
\end{tabular}

En cuanto a la distribución por sexos, 28 (39\%) pacientes correspondieron al sexo femenino frente a $43(60 \%)$ correspondientes al sexo masculino, lo cual representa una relación de 1,5:1 de varones respecto a las niñas.

Entre las manifestaciones clínicas, las lesiones cutáneas estuvieron presentes de manera aislada o en combinación con otros síntomas en 46 casos (64\%); seguidas por los síntomas respiratorios en 38 casos (53\%); y los gastrointestinales en 30 casos $(42,2 \%)$ tal como se presenta en la Figura 1. 


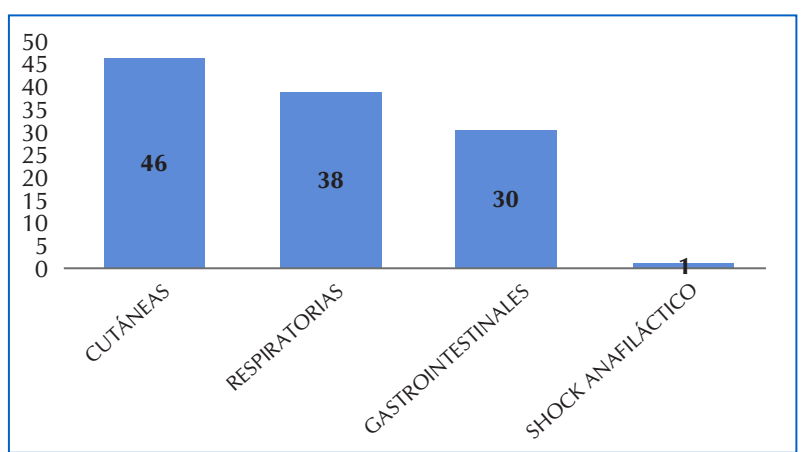

Figura 1. Frecuencia de signos y síntomas de pacientes pediátricos con diagnóstico de alergia alimentaria del Hospital de Clínicas FCM-UNA. Años 2006-2009

Del total de pacientes $31(43.66 \%)$ se presentó con síntomas únicos: 15/71 (21\%) con lesiones cutáneas, 8/71 (11\%) con síntomas gastrointestinales y 8/71 (11\%) con síntomas respiratorios.

La asociación de síntomas cutáneos y respiratorios fue encontrada en 17 casos (24\%); mientras que la asociación de síntomas gastrointestinales y cutáneos fue hallada en 9 casos (12\%) y la asociación de síntomas gastrointestinales y respiratorios en 8 casos $(11 \%)$. La triple asociación de síntomas cutáneos, respiratorios y gastrointestinales fue hallada en 5 casos (7\%). Adicionalmente, se constató escasa ganancia de peso en 9 niños (12\%).

En cuanto a los alérgenos alimentarios, en la Figura 2 se puede observar que la leche estuvo involucrada con el diagnóstico de alergia alimentaria en 52 (73\%), en tanto que el huevo en $33(46 \%)$ pacientes, el trigo en 7 casos $(9,8 \%)$, la soja en $6(8,5 \%)$ casos y el gluten en 5 casos $(7 \%)$; en menor proporción se detectó alergia a golosinas, el maní, el maíz y los embutidos con 2 casos $(2,8 \%)$ cada uno y finalmente la sandía, el tomate y condimentos con 1 caso $(1,4 \%)$ respectivamente.

Se registró un único alérgeno en 46 casos $(64,7 \%)$, dos alérgenos en 16 casos (22,5\%) y en menor frecuencia, a mayor cantidad de alergenos (Tabla 2).

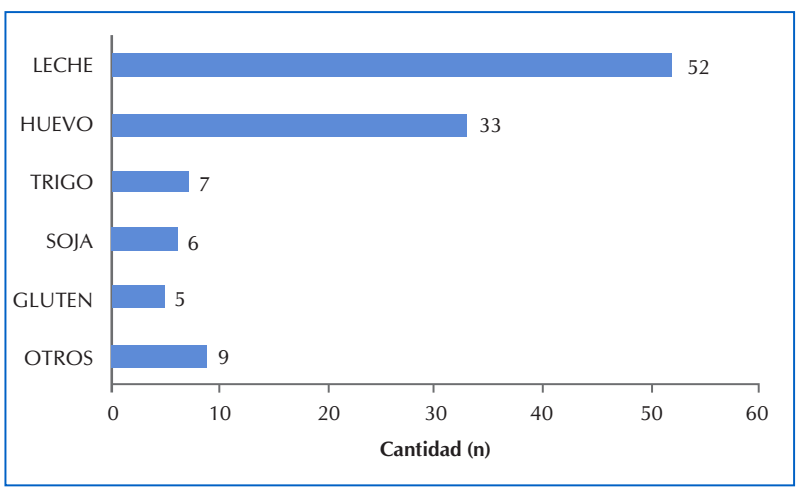

Figura 2. Tipo de alimento involucrado con alergias alimentarias en pacientes pediátricos del Hospital de Clínicas FCM-UNA. Años 2006-2009

Tabla 2. Cantidad de alimentos involucrados con alergias alimentarias en pacientes pediátricos del Hospital de Clínicas FCM-UNA. Años 2006-2009

\begin{tabular}{lcc}
\hline Cantidad de alérgenos & $\mathbf{n}$ & $\mathbf{\%}$ \\
\hline Alimento único & 46 & 64,8 \\
2 alimentos & 16 & 22,5 \\
3 alimentos & 5 & 7,0 \\
$\geq 4$ alimentos & 4 & 5,6 \\
\hline Total & 71 & 100 \\
\hline
\end{tabular}

Como alérgeno único, se registró a la leche en 31 casos $(43,6 \%)$, seguido por el huevo en 12 casos $(16,9 \%)$. La asociación de leche y huevo se encontró en 12 casos $(16,9 \%)$ y como alérgenos asociados a otros alimentos en 18 casos $(25,3 \%)$.

\section{DISCUSIÓN}

El presente trabajo presenta una descripción de casos de alergia alimentaria en una población pediátrica en la que se observan la frecuencia, las manifestaciones clínicas y los alergenos alimentarios involucrados.

La frecuencia de alergia alimentaria hallada coincide con estudios realizados por Smaldini et al. ${ }^{(5)}$ así como la mayor parte de los estudios sobre alergia alimentaria en la población occidental ${ }^{(12)}$.

En cuanto a las edades, este estudio también coincidió con estudios realizados por Stanley et al. ${ }^{(13)}$, siendo la población menor a 2 años la más afectada, y se halló que la edad promedio de 9 meses es ligeramente inferior a los 11 meses que reportan otros autores ${ }^{(14,15)}$. 
En este estudio se constató una ligera predominancia en el sexo masculino con una relación cercana a 3:2 frente al sexo femenino, lo cual contrasta con lo hallado por Aguilar-Jasso ${ }^{(16)}$ quien encontró predominio en el sexo femenino. La realización de más estudios podría respaldar la idea de que el sexo masculino representa un factor de riesgo para el desarrollo de alergias alimentarias como informan Palli-Scholl et al. ${ }^{(17)}$. En otros estudios como el de Venero-Fernández et al. ${ }^{(7)}$ sin embargo, no hallaron diferencias significativas con respecto al sexo.

La manifestación clínica más frecuente en nuestro estudio fueron las lesiones cutáneas con una frecuencia similar a lo publicado por Venter et al..$^{(18)}$ en tanto que los síntomas respiratorios fueron mencionados en la mitad de los niños. Estas manifestaciones se presentaron en forma aislada en la tercera parte de los pacientes estudiados y en el resto mostraron síntomas asociados; siendo las más detectadas las asociaciones gastrointestinales y cutáneas y de síntomas gastrointestinales y respiratorios, coincidente con lo encontrado en revisiones similares ${ }^{(19)}$.

La heterogeneidad de los síntomas nos hace reflexionar que la alergia es una entidad única, pero con diferentes manifestaciones, como rinitis, urticaria, diarrea o constipación, entre otros, sobre todo cuando se reproducen con la existencia de relación temporal entre la aparición de estos síntomas y la introducción o consumo de ciertos alimentos.

Las manifestaciones clínicas, en especial cutáneas, nos sugiere relacionar a la alergia alimentaria con otras entidades, en especial con la dermatitis atópica, que llega a coexistir con la alergia alimentaria en $35-71 \%$ de los $\operatorname{casos}^{(20)}$ según lo descrito por el NIAD (National Institute of Allergy and Infectious Diseases), en Estados Unidos.

El shock anafiláctico es una manifestación grave la alergia alimentaria ${ }^{(21)}$. En contraposición con estudios realizados en Estados unidos donde se reportan hasta un $38,7 \%$ de anafilaxia ${ }^{(22)}$, en nuestro trabajo existió el antecedente de shock anafiláctico en un solo paciente.
Las repercusiones en cuanto al crecimiento y desarrollo del niño con alergia alimentaria ya fueron descritas por Stanley et al. ${ }^{(8)}$. En esta muestra se detectó escasa ganancia ponderal en el 12,6\% de la población, ligeramente inferior al $25 \%$ de la población descrita por Christie et al. ${ }^{(8)}$.

En este estudio, la leche de vaca y el huevo fueron los principales alérgenos reportados. En estudios realizados por Mullins et al. ${ }^{(14)}$, se mencionan cifras de $2,7 \%$ para la leche de vaca y $8,8 \%$ para el huevo. En otro trabajo, realizado en Argentina, se identifica a la leche de vaca como el principal alérgeno, sin que se tengan cifras estadísticas reales. En este trabajo la leche de vaca representó el $73 \%$ del total de la muestra, mientras que el huevo se encontró en $46 \%$ de los casos como alérgeno. Otros alimentos como el maní o los mariscos no se reportan como importantes alérgenos en nuestra muestra. Esto se debe probablemente a que dichos alimentos no son consumidos en forma habitual por la población general y menos por los lactantes, quienes son los más susceptibles al desarrollo de alergias alimentarias. En cambio, la leche de vaca y el huevo son importantes componentes de la alimentación de nuestra población ya sea al ser consumidos como tales, o a través de sus derivados. Es por ello que se debe insistir en la importancia de realizar dietas excluyentes en forma apropiada, teniendo en cuenta que estos alimentos pueden ser encontrados como ingredientes de alimentos procesados ${ }^{(23)}$. Llama la atención que, del total de alérgenos posibles, sólo la leche de vaca y el huevo se encontraron en una proporción importante. Ninguno de los restantes alérgenos reportados superó el $10 \%$ de la población estudiada, y en algunos casos (como la sandía, el tomate o los condimentos) solo se reportaron casos aislados.

Si bien en la mayoría de los casos la alergia alimentaria se presentó con un único alérgeno, existe un porcentaje importante $(36 \%)$ de alergias alimentarias múltiples, cifra similar a la que informa Plaza-Martín (un tercio de los casos) ${ }^{(13)}$. Esto representa un verdadero desafío a la hora de elaborar y cumplir las dietas de exclusión y determina que probablemente un niño con esta condición tendrá medidas antropométricas inferiores a un niño sin alergia alimentaria. 
Una limitante por el diseño del estudio constituye el déficit de datos para valorar factores de riesgo como los antecedentes familiare ${ }^{(24)} \mathrm{o}$ las características de la alimentación, como tipo de lactancia e introducción de alimentos complementarios ${ }^{(25)}$, ya que varios estudios proponen que el microbiota intestinal inducida por la alimentación pudiera prevenir o predisponer la emergencia de alergias alimentarias ${ }^{(26)}$ aunque el mecanismo continúa siendo objeto de investigación ${ }^{(27,28)}$. También es necesario considerar elementos relacionados con el medio ambiente además de los factores alimentarios, así como la predisposición genética ${ }^{(29)}$. Es sabido, que los estudios de cohorte podrán aportar más información sobre estos factores.

Por otra parte, se menciona como limitante la cantidad final de pacientes elegibles para el estudio y por ello, evidentemente es importante trabajar de manera multicéntrica con centros de referencia como es nuestro servicio, a fin de aumentar la muestra de estudio para el grupo etario pediátrico, a fin de conocer cuál es la prevalencia y cómo son las características de las alergias alimentarias a nivel nacional.

\section{CONCLUSIONES}

Los lactantes son los más afectados por las alergias alimentarias en más de dos tercios de los casos, seguido por los niños en edad preescolar. En los escolares y adolescentes la alergia alimentaria es menos frecuente.

\section{REFERENCIAS}

1. Noguera F, André L, Freitas C, Hallison C. Food allergy: is desensitization an effective treatment strategy in patients with peanut allergy?. Revista de Medicina da UFC 2019; 59:33-39.

2. Iweala OI, Burks AW. Food Allergy: Our Evolving Understanding of Its Pathogenesis, Prevention, and Treatment. Curr Allergy Asthma Rep. 2016; 16(5):37. doi: http://dx.doi.org/10.1007/s11882-016-0616-7
Existe un ligero predominio del sexo masculino para el desarrollo de alergias alimentarias.

Las manifestaciones más frecuentes son por orden de frecuencia las cutáneas en más de dos tercios de los casos, respiratorias en la mitad de los niños y gastrointestinales en un tercio de los pacientes, ya sea en forma solitaria o en combinación estos y otros alérgenos. El shock anafiláctico es un hallazgo poco frecuente.

Los principales alérgenos involucrados en las alergias alimentarias en nuestra población son la leche de vaca y el huevo en más de la mitad de los casos.

La alergia alimentaria única fue la más frecuente, 1/4 de los pacientes reacciona a dos alérgenos y en menor proporción a 3 o más alérgenos.

\section{CONTRIBUCIÓN DE TODOS LOS AUTORES}

Marco Antonio González Miltos: Concepción y diseño del trabajo, elaboración del protocolo, toma de muestra, sistematización de datos, redacción del manuscrito.

Ricardo Meza: Apoyo en la concepción y diseño del trabajo, corrección de redacción del manuscrito.

Susana Sánchez Bernal: Apoyo en la redacción del manuscrito, procesamiento de datos, corrección, edición y ajuste del manuscrito.

3. Hill DA, Grundmeier RW, Ram G, Spergel, JM. The epidemiologic characteristics of healthcare providerdiagnosed eczema, asthma, allergic rhinitis, and food allergy in children: a retrospective cohort study. BMC P e d i a t r i c s $2016 ; 16(1): 2-8$. d o i : http://dx.doi.org/10.1186/s12887-016-0673-z

4. Peters RL, Koplin JJ, Gurrin LC, Dharmage SC, Wake M, Ponsonby AL, et al. The prevalence of food allergy and other allergic diseases in early childhood in a population- 
based study: HealthNuts age 4-year follow-up. J Allergy Clin Immunol 2017; 140(1):145-153. doi: http://dx.doi. org/10.1016/j.jaci.2017.02.019

5. Smaldini P, Orsini ML. Avances en las inmunoterapias para alergias alimentarias. Acta Bioquímica Clínica Latinoamericana. 2016;50(1):61-75.

6. Sicherer SH, Allen K, Lack G, Taylor SL, Donovan SM, Oria M. Critical Issues in Food Allergy: A National Academies Consensus Report. Pediatrics. 2017; 140(2):110. doi: http://dx.doi.org/10.1542/peds.2017-0194

7. Rojas AR, Quezada LA. Relación entre dermatitis atópica y alergia alimentaria. Rev. chil. pediatr. [Internet]. 2013 [citado 2021 Nov 11]; 84(4):438-450. Disponible en: http://www.scielo.cl/scielo.php?script=sci_arttext\&pid=S 0370-41062013000400012\&lng=es doi: http://dx.doi.org /10.4067/S0370-41062013000400012

8. Venero-Fernández SJ, Bringues-Menzie V, Méndez-Rotger MT, Fernández-Casamayor A, Urbina-Reinaldo J, ÁlvarezCastelló M, et al. Prevalencia, incidencia y factores asociados con reacción adversa a alimentos en infantes cubanos. Estudio de cohorte de base poblacional. Rev. alerg. Méx [Internet]. 2018 [citado 2021 Nov 11]; 65(2):117-127. Disponible en: http://www.scielo.org.mx/scielo.php?script=s ci_arttext\&pid=S2448-91902018000200117\&lng=es doi: https://doi.org/10.29262/ram.v65i2.301

9. Coronel Carvajal C. Factores atópicos asociados a la sibilancia recurrente en el menor de cinco años. AMC [Internet]. 2018 [citado 2021 Nov 11]; 22(4):417-431. Disponible en: http://scielo.sld.cu/scielo.php?script=s ci_arttext\&pid=S1025-02552018000400417\&lng=es.

10. Savage J, Sicherer S, Wood R. The Natural History of Food Allergy. J Allergy Clin Immunol Pract. 2016; 4(2):196203. doi: http://dx.doi.org/10.1016/j.jaip.2015.11.024

11. Du Toit G, Sampson HA, Plaut M, Burks AW, Akdis CA, Lack G. Food allergy: Update on prevention and tolerance. J Allergy Clin Immunol. 2018; 141(1):30-40. doi: http://dx.doi.org/10.1016/j.jaci.2017.11.010

12. Toro-Monjaraz EM, Ramírez-Mayans JA. Alergia alimentaria en pediatría. Rev Gastroenterol Méx. 2016; 81(Supl 1):32-34.

13. Stanley S. Diet in Food Allergic Children: Impact of Food Allergy on Growth and Food Group Intake. Tesis publicada por ProQuest Dissertations Publishing. Incahn School of Medicina en Mount Sianai. Michigan; 2019.

14. Plaza-Martin, AM. (2016). Alergia alimentaria en la edad pediátrica, conceptos actuales. Anales de Pediatría. 2016; 85(1): 50.e1-50.e5. doi: http://dx.doi.org/10.1016/j.anpedi.2016.01.017
15. Peters RL, Koplin JJ, Gurrin LC, Dharmage SC, Wake $\mathrm{M}$, Ponsonby A-L, et al. The prevalence of food allergy and other allergic diseases in early childhood in a populationbased study: HealthNuts age 4-year follow-up Journal of Allergy and Clinical Immunology. 2017; 140(1):145-153. doi:http://dx.doi.org/10.1016/jjaci201702019

16. Aguilar-Jasso D. Perfil clínico de pacientes pediátricos con diagnóstico de alergia alimentaria en el noroeste de México. Revista Alergia México. 2018; 65:233-241.

17. Patel N, Herbert L, Green TD. The emotional, social, and financial burden of food allergies on children and their families. Allergy Asthma Proc. 2017; 38(2):88-91. doi: http://dx.doi.org/10.2500/aap.2017.38.4028

18. Venter C, Palumbo MP, Sauder KA, Glueck DH, Liu AH, Yang IV, et al. Incidence and timing of offspring asthma, wheeze, allergic rhinitis, atopic dermatitis, and food allergy and association with maternal history of asthma and allergic rhinitis. World Allergy Organ J. 2021; 14(3):1-14. doi: http://dx.doi.org/10.1016/j.waojou.2021.100526

19. Bingol A, Uygun DFK, Akdemir M, Erengin $H$, Buyuktiryaki B, Sackesen C, Bingol G, et al. Clinical phenotypes of childhood food allergies based on immune mechanisms: A multicenter study. Allergy Asthma Proc 2021;42(3):e86-e95. doi: http://dx.doi.org/10.2500/aap.2 021.42.210005

20. Sooyoung L. IgE-mediated food allergies in children: prevalence, triggers, and management. Korean J Pediatr. 2017; 25:99-105. doi: http://dx.doi.org/10.3345/kjp .2017.60.4.99

21. Sooyoung L. IgE-mediated food allergies in children: prevalence, triggers, and management. Korean J Pediatr. 2017; 25:99-105. doi: http://dx.doi.org/10.3345/kjp.20 17.60.4.99

22. Renz H, Allen KJ, Sicherer SH, Sampson HA, Lack G, Beyer K, et al. Food allergy. Nat Rev Dis Primers. 2018; 4:17098. doi: http://dx.doi.org/10.1038/nrdp.2017.98

23. Warren CM, Turner PJ, Chinthrajah RS, Gupta RS. Advancing Food Allergy Through Epidemiology: Understanding and Addressing Disparities in Food Allergy Management and Outcomes. J Allergy Clin Immunol Pract. 2021; 9(1):110-118. doi: http://dx.doi.org/1 0.1016/j.jaip.2020.09.064

24. Mastrorilli C, Caffarelli C, Hoffmann-Sommergruber K. Food allergy and atopic dermatitis: Prediction, progression, and prevention. Pediatr Allergy Immunol. 2017; 28(8):831840. doi: http://dx.doi.org/10.1111/pai.12831 
25. Heine RG. Food Allergy Prevention and Treatment by Targeted Nutrition. Ann Nutr Metab. 2018; 72(3):33-45. doi: http://dx.doi.org/10.1159/000487380

26. Ipci K, Alt?ntoprak N, Muluk NB, Senturk M, Cingi C. The possible mechanisms of the human microbiome in allergic diseases. Eur Arch Otorhinolaryngol 2017; 274(2):617-626. doi: http://dx.doi.org/10.1007/s00405-0164058-6

27. Han P, Gu JQ, Li LS, Wang XY, Wang HT, Wang Y, et al. The Association Between Intestinal Bacteria and Allergic Diseases-Cause or Consequence? Front Cell Infect Microbiol. 2021; 11:650893. doi: http://dx.doi.org/10. 3389/fcimb.2021.650893
28. Marrs T, Sim K. Demystifying Dysbiosis: Can the Gut Microbiome Promote Oral Tolerance Over IgE-mediated Food Allergy? Curr Pediatr Rev. 2018; 14(3):156-163. doi: http://dx.doi.org/10.2174/1573396314666180507120424

29. Boutin RCT, Sbihi H, McLaughlin RJ, Hahn AS, Konwar KM, Loo RS, et al. Composition and Associations of the Infant Gut Fungal Microbiota with Environmental Factors and Childhood Allergic Outcomes. Cell Reports Medicine 2021; 2:1-13. doi: http://dx.doi.org/ 10.1128/mBio.03396-20 